\title{
Theorie der stoßfreien Plasmasäule unter Berücksichtigung des Wandverlustes
}

\author{
G. ECKER \\ Institut für Theoretische Physik der Universität Bochum * \\ und H. KANNE \\ Institut für Theoretische Physik der Universität Bonn \\ (Z. Naturforschg. 21 a, 719_725 [1966] ; eingegangen am 25. Februar 1966)
}

\begin{abstract}
The collision-free plasma column has been investigated without a magnetic field in the normal and subnormal region neglecting wall losses. This neglection simplifies the analysis appreciably since it allows to describe the electrons by a Boltzmans distribution. However, in the subnormal region and in strong magnetic fields the wall loss is a decisive factor. Therefore our investigation extends the theory including this effect.

Starting from the transport equations particle density and potential distributions as well as the current-voltage characteristic are found by numerical integration.

With increasing magnetic field the particle density profiles grow wider whereas the potential distribution grows flatter. These effects are particularly pronounced in the subnormal region. In this region the $I / V$-characteristic shows a negative slope, which for a given current decreases witk. increasing magnetic field.
\end{abstract}

Die theoretische Analyse der zylindrischen Plasmasäule ist von praktischem Interesse für die Anwendungen im Bereich der Gasentladungen und von prinzipiellem Interesse für das Verständnis der Elementarprozesse in der Plasmaphysik. Für die stoßbestimmte Säule, deren Ionenweglänge wesentlich kleiner ist als der Säulenradius $\left(l_{+} \ll R\right)$ liegen zahlreiche Untersuchungen des stationären und nichtstationären Verhaltens mit und ohne Berücksichtigung von Magnetfeldern vor. Demgegenüber ist die Theorie der stoßfreien Säule $\left(l_{+} \geqq R\right)$ ohne Magnetfeld im wesentlichen nur in zwei Arbeiten behandelt ${ }^{1,2}$. Darüberhinaus wurde der Einfluß eines schwachen, transversalen Magnetfeldes untersucht ${ }^{3}$.

Die Untersuchung von Langmuir ${ }^{1}$ setzt für die Elektronen die BoltzmanN-Verteilung voraus und nimmt an, daß die Ionen mit vernachlässigbarer Geschwindigkeit erzeugt werden und radial frei zur Wand fallen. Unter diesen Umständen leitet LANGMUIR eine Integrodifferentialgleichung für das Potential als Funktion des Ortes her. Im Fall der „normalen Säule“, deren Deвye-Länge gegenüber dem Säulenradius klein ist $\left(l_{\mathrm{D}} \ll R\right)$, gibt LANGMuir eine befriedigende analytische Näherungslösung dieser Gleichung an.

SELF ${ }^{2}$ löst die Langmuirsche Integrodifferentialgleichung mit maschinellen Mitteln. Die Ergebnisse stimmen im „normalen Bereich“ $\left(l_{\mathrm{D}} \ll R\right)$ mit denen

* Vorläufige Anschrift: Bonn, Wegelerstr. 10.

1 L. Tonss u. I. Langmuir, Phys. Rev. 34, 876 [1929]. von Langmuir überein. Darüberhinaus erfassen sie den "subnormalen Bereich“ $\left(l_{\mathrm{D}} \cong R\right)$.

Die Überlagerung eines transversalen, schwachen Magnetfeldes ändert die Elektronendichteverteilung und die Ionenbewegung. Die Änderung der Elektronendichteverteilung läßt sich durch die Berücksichtigung des Hall-Potentials im Exponenten der Boltzmann-Verteilung erfassen. Die Ionenbewegung kann angenähert als radialer freier Fall von einem verschobenen Potential-Zentrum aus beschrieben werden ${ }^{3}$.

\section{Das Problem}

Die beiden entscheidenden Voraussetzungen der im Vorgang zitierten Theorien sind die geradlinige stoßfreie Ionenbewegung und die Boltzmann-Verteilung der Elektronen. Die Annahme für die Ionenbewegung kann durch die Wahl des Gefäßradius bei jeder Feldstärke des äußeren Magnetfeldes gesichert werden. Demgegenüber gibt es in allen Fällen einen Wandbereich der Plasmasäule, wo die Voraussetzung der BoltzmanN-Verteilung der Elektronen nicht gerechtfertigt ist. Dieser Bereich umfaßt das Gebiet, in dem der Wandverluststrom vergleichbar ist mit der mittleren Elektronen-Fluktuation. Die Abweichung von der Boltzmann-Verteilung der Elektronen wird sich daher mit zu-

2 S. A. Self, Phys. Fluids 6, 1762 [1963].

3 G. Ecker u. H. Kanne, Phys. Fluids 7, 1834 [1964]. 
nehmender Subnormalität und mit zunehmendem longitudinalen Magnetfeld immer stärker bemerkbar machen.

Die folgende Analyse trägt dem Elektronenwandverlust angenähert Rechnung und bestimmt seine Auswirkung auf die Säuleneigenschaften.

\section{Das Modell}

Wir untersuchen ein stationäres zylindrisches Plasma mit unendlicher axialer Ausdehnung. Das Plasma ist axial homogen und enthält nur eine Neutralteilchenart, eine Art positiver Ionen und Elektronen. Wir beschränken uns auf einen Strombereich, in dem die Annahme konstanter Temperatur und schwacher Ionisation gerechtfertigt ist. Die Ladungsträger werden erzeugt durch ElektronenNeutralteilchen-Stoß und vernichtet durch Wandrekombination. Die Elektronentemperatur ist konstant und von der Gastemperatur verschieden. Ein etwa vorhandenes Magnetfeld liege in axialer Richtung.

\section{Formulierung des Problems}

Zur Beschreibung unseres Systems verwenden wir die Erhaltungssätze für Teilchenzahlen und Impulse.

Die Teilchenerhaltung der Ionen drückt sich im stationären Zustand in der Form

$$
\nabla \cdot \Gamma_{+}=n_{-} / \tau
$$

aus, wenn $\Gamma$ der Teilchenstrom, $n$ die Teilchendichte und $1 / \tau$ der Erzeugungskoeffizient ist. Die Impulsbilanz des Einzelteilchens ergibt nach einfacher Integration für die Geschwindigkeit $v_{+}$der bei $r=\varrho$ erzeugten Ionen

$$
v_{+}(\varrho, r)=\sqrt{\left(2 e / m_{+}\right)[U(\varrho)-U(r)]},
$$

wobei $U$ das Potential, $m$ die Teilchenmasse und $e$ die Elementarladung bedeutet. Aus (1) folgt die radiale $(r)$ Ionenstromdichte

$$
\Gamma_{+r}(r)=\int_{0}^{r} \frac{n_{-}(\varrho)}{\tau} \frac{o}{r} \mathrm{~d} \varrho
$$

und aus (1) und (2) die Ionendichte

$$
n_{+}(r)=\int_{0}^{r} \frac{(1 / \tau) n_{-}(\varrho)}{v_{+}(\varrho, r)} \frac{\varrho}{r} \mathrm{~d} \varrho .
$$

Diese bereits von LangmuIR angegebenen Gleichungen setzen voraus, daß die Ionen mit vernachlässig- barer Geschwindigkeit erzeugt werden und sich dann radial geradlinig und stoßfrei bewegen. Die Feldstärke $B$ eines äußeren axialen Magnetfeldes wird durch diese Annahme auf Werte beschränkt, die den Krümmungsradius der Ionenbahnen wesentlich größer als den Säulenradius lassen. Die Teilchenerhaltung der Elektronen beschreibt die Beziehung

$$
\nabla \cdot \Gamma_{-}=n_{-} / \tau \text {. }
$$

Die stationäre Impulstransportgleichung der Elektronen lautet

$$
\begin{aligned}
m_{-}\left(\Gamma_{-} / n_{-}\right) & \cdot \nabla\left(\Gamma_{-} / n_{-}\right)=e\left[\nabla U-\left(\Gamma_{-} / n_{-}\right) \times B\right] \\
& -v m_{-}\left[\left(\Gamma_{-} / n_{-}\right)-\left(\Gamma_{0} / n_{0}\right)\right] .
\end{aligned}
$$

Entsprechend dem allgemeinen Gebrauch ist hier im Stoßglied der Mittelwert über das Produkt von Stoßfrequenz $v$ und Geschwindigkeit durch das Produkt der Mittelwerte von Stoßfrequenz und der Geschwindigkeit ersetzt worden. Die Elektronen-Ionenwechselwirkung ist bei der angenommenen schwachen Ionisation unbedeutend. In der Beziehung (6) kann der Trägheitsterm auf der linken Seite und die mittlere Neutralgasgeschwindigkeit im letzten Glied der rechten Seite vernachlässigt werden, solange $\left(l_{-} / R\right)^{2}$ $\ll T_{-} / T_{0}$. Ferner wird im Anhang begründet, daß die skalare Approximation des Drucktensors $p_{-}$erlaubt ist. Damit gilt für unser Modell

$$
\left(v m_{-} / e\right) \Gamma_{-}=n_{-} \nabla U-\Gamma_{-} \times B-\left(k T_{-} / e\right) \nabla n_{-} .
$$

Unter Berücksichtigung der Rotationssymmetrie folgt aus dieser Gleichung für die radiale Stromkomponente

$$
\Gamma_{-r}=D_{-}\left(\frac{e n_{-}}{k T_{-}} \frac{\mathrm{d} U}{\mathrm{~d} r}-\frac{\mathrm{d} n_{-}}{\mathrm{d} r}\right),
$$

wo der Diffusionskoeffizient $D_{-}$und die Beweglichkeit (im Magnetfeld) $\mu_{-}{ }^{*}$ durch

$$
\begin{aligned}
D_{-} & =\left(k T_{-} / e\right) \frac{e /\left(v m_{-}\right)}{1+\left[e B /\left(v m_{-}\right)\right]^{2}} \\
& =\left(k T_{-} / e\right) \mu_{-}{ }^{*}
\end{aligned}
$$

gegeben ist. Die elementare BoLtzmann-Verteilung für die Elektronen folgt aus Gl. (8), wenn $\Gamma_{-r}$ gegen die einzelnen Glieder der rechten Seite vernachlässigt werden kann.

Aus den Gln. (1) und (5) folgt die Stationaritätsbeziehung für den elektrischen Strom

$$
\nabla \cdot\left(\Gamma_{+}-\Gamma_{-}\right)=0
$$

und damit unter Berücksichtigung der axialen Homogenität, der Rotationssymmetrie und der Stromlosig- 
keit an der Wand die Kongruenzrelation

$$
\Gamma_{+r}=\Gamma_{-r} .
$$

Durch Einführung der Beziehungen (3) und (8) in (11) ergibt sich ein Zusammenhang zwischen dem elektrischen Potential und der Elektronendichte.

Eine zweite Beziehung zwischen diesen beiden Größen liefert unter Verwendung der Gl. (4) die Porsson-Gleichung

$$
\Delta U=-\left(e / \varepsilon_{0}\right)\left(n_{+}-n_{-}\right) .
$$

Unser Problem wird also durch das folgende Integrodifferentialgleichungssystem beschrieben

$$
\begin{aligned}
& \int_{0}^{r} \frac{n_{-}(\varrho)}{\tau} \frac{\varrho}{r} \mathrm{~d} \varrho=D_{-}\left[\frac{e n_{-}(r)}{k T_{-}} \frac{\mathrm{d} U(r)}{\mathrm{d} r}-\frac{\mathrm{d} n_{-}(r)}{\mathrm{d} r}\right], \\
& \frac{\varepsilon_{0}}{e r} \frac{\mathrm{d}}{\mathrm{d} r} r \frac{\mathrm{d}}{\mathrm{d} r} U(r) \\
& =n_{-}(r)-\int_{0}^{r} \frac{n_{-}(\varrho) / \tau}{\sqrt{\left(2 e / m_{+}\right)[U(\varrho)-U(r)]}} \frac{\varrho}{r} \mathrm{~d} \varrho .
\end{aligned}
$$

Die zentrale Elektronendichte $n_{-}(0)=n_{\mathrm{c}}$ und der Diffusionskoeffizient $D_{\text {- }}$ sind durch die Stromstärke $I$ und das Magnetfeld $B$ festgelegt.

Aus der Mannigfaltigkeit der Lösungen von (13) und (14) wird die uns interessierende durch die Randbedingungen

$$
\begin{array}{cl}
r=0: & U=0 ; \quad \mathrm{d} U / \mathrm{d} r=0 ; \\
& n_{-}=n_{\mathrm{c}} ; \quad \mathrm{d} n_{-} / \mathrm{d} r=0 ; \\
r=r_{\mathrm{w}}: \quad \Gamma_{+r}=\Gamma_{-r}=n_{-} \bar{v}_{-} / 4
\end{array}
$$

ausgewählt $\left(\bar{v}_{-}=\right.$mittlere Elektronengeschwindigkeit). Der Erzeugungskoeffizient $1 / \tau$ ergibt sich als der Eigenwert des Problems.

Führen wir zur Vorbereitung der Lösung im Nachgang zu LANGmuir anstelle der radialen Koordinate $r$ das Potential $U$ als unabhängige Variable ein und verwenden die Abkürzungen

$\eta=-e U /\left(k T_{-}\right), n_{-}=n_{\mathrm{c}} n, n_{+}=n_{\mathrm{c}} f$,

$x=\lambda r, l_{\mathrm{D}}{ }^{\mathrm{c}}=\sqrt{\varepsilon_{0} k T_{-} /\left(e^{2} n_{\mathrm{c}}\right)}$,

$\lambda=2 \sqrt{\pi}\left(m_{-} / m_{+}\right)$,

$a=\left(\lambda l_{D}{ }^{c}\right)^{2}, \beta=1 /\left(\lambda^{2} \tau D_{-}\right)$,

$\gamma=(1 / \tau) \sqrt{m_{+} /\left(2 k T_{-}\right)}$,

so ergeben sich die dimensionslosen Beziehungen

$$
\begin{gathered}
\frac{\mathrm{d}}{\mathrm{d} \eta} \ln \frac{-\dot{x} /(n x)}{1+\dot{x} / n}=\frac{\beta \dot{x}^{2}}{1+\dot{x} / n}, \\
\left(\alpha / \dot{x}^{2}\right) \frac{\mathrm{d}}{\mathrm{d} \eta} \ln (\dot{x} / x)=n-\frac{1}{x} \int_{0}^{\pi / 2} \mathrm{~d} \psi\left[n(s) \sqrt{s} \frac{\mathrm{d}}{\mathrm{d} s} x^{2}(s)\right] \\
\text { mit } \quad s=\eta \sin ^{2} \psi
\end{gathered}
$$

$$
\begin{aligned}
& x=0: \quad \eta=0 ; \quad \dot{x}=\infty ; \quad n=1 ; \quad \dot{n}=0 ; \\
& x=x_{\mathrm{w}}: \quad 1+\dot{n} / n+\beta / \gamma \dot{x}=0 .
\end{aligned}
$$

Der Punkt bezeichnet hier die Ableitung nach $\eta$.

Die Einführung von $U$ als unabhängiger Variable beseitigt an der Stelle $\varrho=r$ die Singularität des Integrals in (14). Die Singularitäten an der Stelle $r=0$ in (13) und (14) werden durch geeignete Reihenentwicklungen behoben.

\section{Lösung}

Wir wählen jetzt Werte für die Parameter $\alpha$ und $\beta$, die den Grad der Subnormalität und die Stärke des Magnetfeldes charakterisieren, und integrieren von den Anfangswerten bei $x=0$ aus bis zu dem Wert $x=x_{\mathrm{w}}$, an dem die Wandbedingung (19 b) erfüllt ist. $x_{\mathrm{w}}$ liefert dann über die Definition (16) den Eigenwert $1 / \tau$ unseres Problems, aus dem wir mit Hilfe der Beziehung

$$
\begin{gathered}
1 / \tau=A\left(\overline{v_{-}} / l_{-}\right) e^{-1 / \Theta}(1+2 \Theta) ; \\
\Theta=k T_{-} /\left(e U_{\mathrm{i}}\right)
\end{gathered}
$$

die Elektronentemperatur $T_{\text {_ }}$ errechnen $\left(U_{\mathrm{i}}=\right.$ Ionisationsenergie).

Da andererseits zwischen Temperatur $T_{-}$und axialer Feldstärke $E$ ein Zusammenhang besteht und der Strom $I$ durch

mit

$$
\begin{gathered}
I=\int_{0}^{R} 2 \pi r \mathrm{~d} r e \mu_{-} E n_{-} \\
\mu_{-}=e /\left(\nu m_{-}\right)
\end{gathered}
$$

gegeben ist, kennen wir die $E / I$-Charakteristik.

\section{Ergebnisse}

Eine analytische Lösung unseres Gleichungssystems, die im Zentrum schnell konvergiert, kann durch die Reihenentwicklungen

$$
x=\sqrt{\eta} \Sigma a_{\nu} \eta^{\nu}, \quad n=\Sigma b_{\nu} \eta^{\nu}, \quad f=\Sigma c_{\nu} \eta^{\nu}
$$

gewonnen werden. Für die ersten Koeffizienten ergibt sich durch Koeffizientenvergleich:

$$
\begin{array}{ll}
a_{0}^{2}\left(a_{0}-1\right)=4 \alpha, & a_{1}=-a_{0} b_{1} \frac{3-2 a_{0}}{24-29 a_{0}}, \\
b_{0}=1, & b_{1}=-1-a_{0}^{2} \beta / 4, \\
c_{0}=a_{0}, & c_{1}=a_{0} b_{1} \frac{11-16 a_{0}}{24-29 a_{0}} .
\end{array}
$$




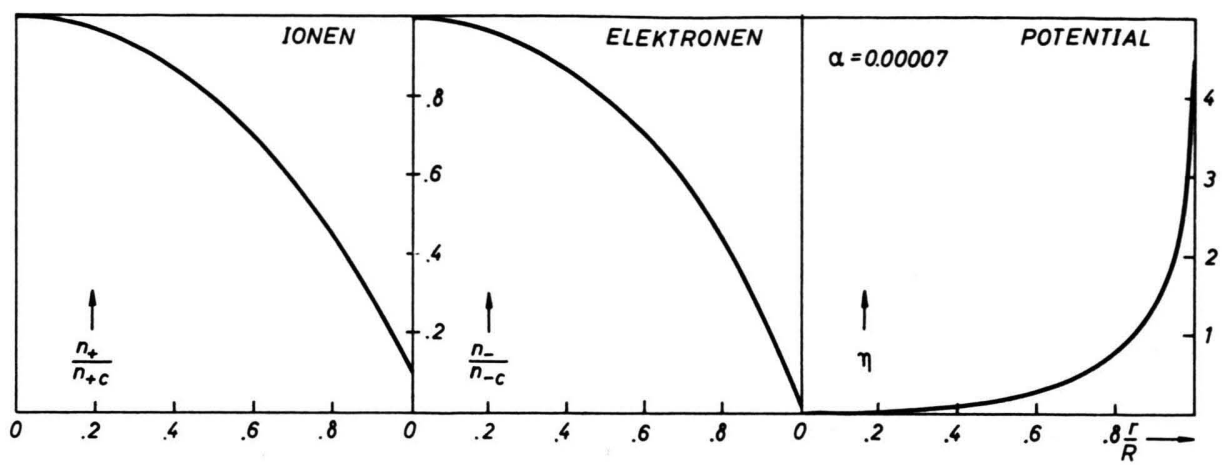

Abb. 1. Trägerdichte- und Potentialverlauf bei kleinem $\alpha$. Die Abhängigkeit von $\beta$ ist so gering, daß sie nicht eingezeichnet wurde.

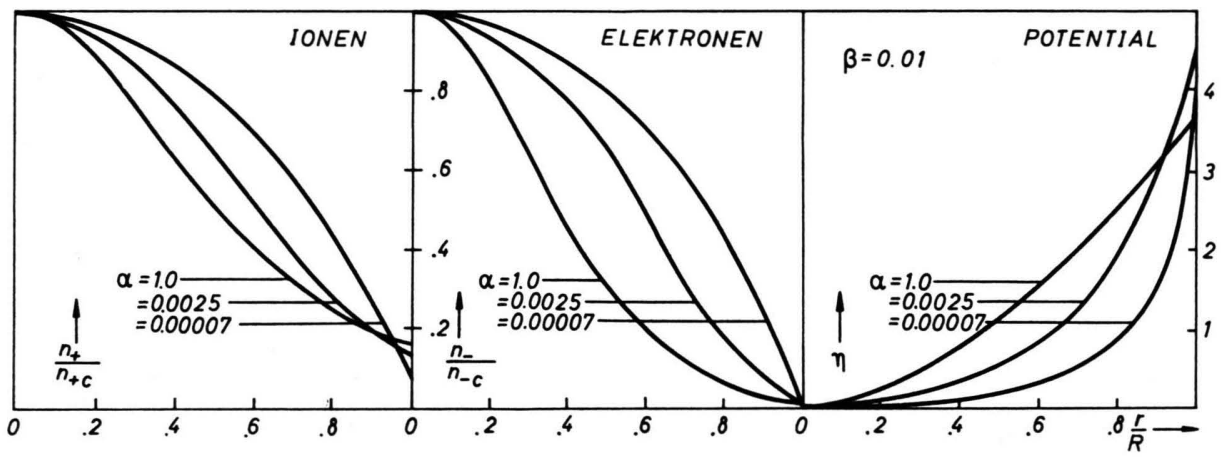

Abb. 2. Trägerdichte- und Potentialverlauf bei großem $\alpha$ in Abhängigkeit von $\beta$. Innerhalh des Parameterbereichs $0 \leqq \beta \leqq 0,01$ ändert sich der Kurvenverlauf nicht mehr.

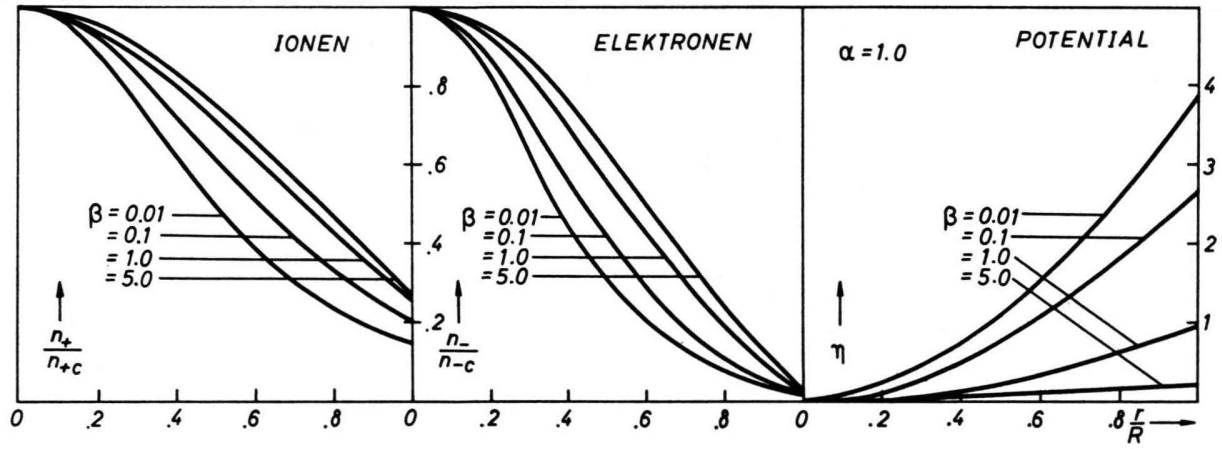

Abb. 3. Trägerdichte- und Potentialverlauf bei festem $\beta$ in Abhängigkeit von $\alpha$. Innerhalb des Parameterbereichs $0 \leqq \alpha \leqq 0,00007$ ändert sich der Kurvenverlauf nicht mehr.

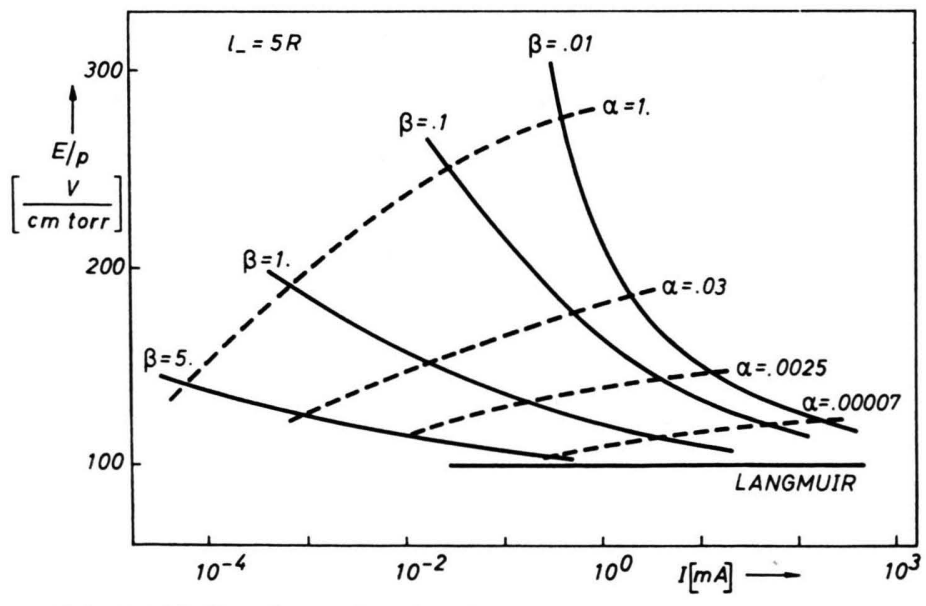

Abb. 4. $E / I$-Charakteristiken bei $l_{-}=5 R$ in Abhängigkeit vom Mağnetfeld.

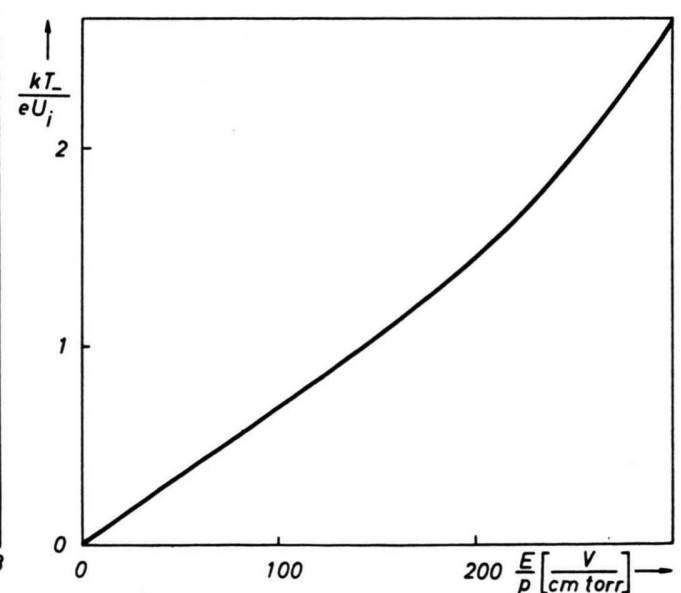

Abb. 6. Abhängigkeit der Elektronentemperatur von der Feldstärke ${ }^{4}$. 


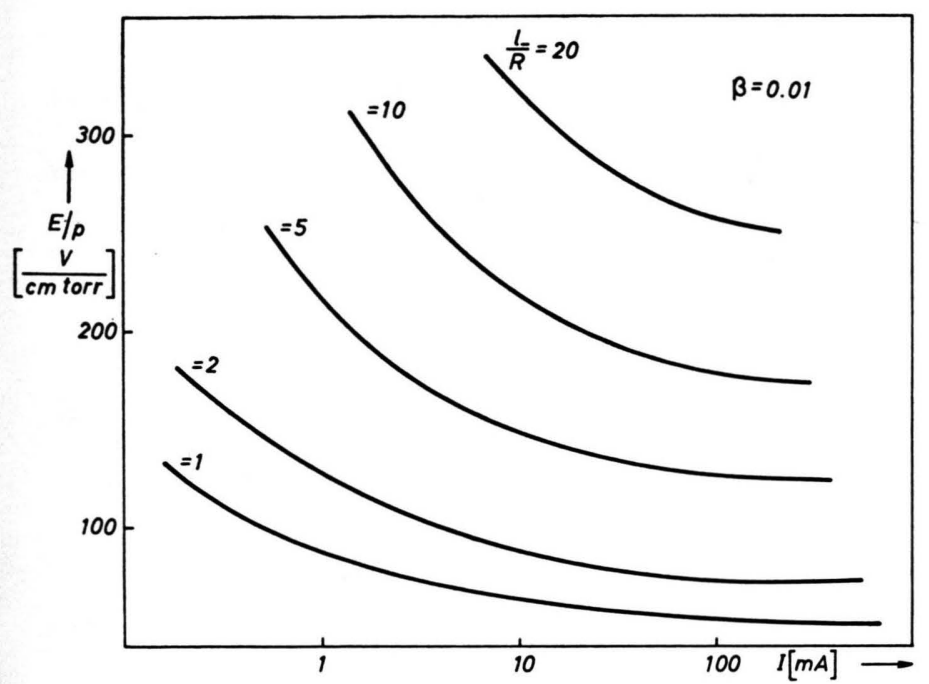

Abb. 5. $E / I$-Charakteristiken bei $\beta=0,01$ in Abhängigkeit von $p R$.

\begin{tabular}{|l|l|l|l|l|l|c|}
\hline \multicolumn{1}{|c|}{$\alpha$} & \multicolumn{1}{c|}{$\beta$} & $l_{\mathrm{D}}^{\mathrm{w}} / R$ & $n_{+\mathrm{c}} / n_{-\mathrm{c}}$ & $x_{\mathrm{w}}$ & $n_{\mathrm{w}}$ & $\eta_{\mathrm{w}}$ \\
\hline 0,00007 & 0,01 & 0,1 & 1,00027 & 0,872 & 0,0106 & $\mathbf{4 , 6 4}$ \\
& 0,1 & & & 0,866 & 0,0105 & $\mathbf{4 , 5 0}$ \\
& 1,0 & & & 0,810 & 0,0101 & 4,18 \\
& 5,0 & & & 0,665 & 0,0070 & 3,07 \\
1,0 & 0,01 & 3,0 & 2,0 & 4,43 & 0,0204 & 3,69 \\
& 0,1 & & & 3,64 & 0,0199 & 2,64 \\
& 1,0 & & & 1,99 & 0,0155 & 0,88 \\
& 5,0 & & & 1,02 & 0,0086 & 0,23 \\
0,00007 & 0,01 & 0,1 & 1,00027 & 0,87 & 0,0106 & 4,64 \\
0,0025 & & 0,4 & 1,01 & 1,22 & 0,0110 & 4,61 \\
0,03 & & 0,9 & 1,1 & 1,96 & 0,0121 & 4,53 \\
1,0 & & 3,0 & 2,0 & 4,43 & 0,0204 & 3,69 \\
0,00007 & 5,0 & 0,1 & 1,00027 & 0,67 & 0,0070 & 3,07 \\
0,0025 & & 0,4 & 1,01 & 0,79 & 0,0071 & 1,67 \\
0,03 & & 0,9 & 1,1 & 0,90 & 0,0073 & 0,92 \\
1,0 & & 3,0 & 2,0 & 1,02 & 0,0086 & 0,23 \\
\hline
\end{tabular}

Tab. 1. Ergebnisse der Rechnung.

Der Koeffizient $a_{0}$ aus der Entwicklung $x(\eta)$ gibt also gleichzeitig das Verhältnis der Trägerdichten im Zentrum $n_{+\mathrm{c}} / n_{-\mathrm{c}}=c_{0} / b_{0}$ an. Dieses Verhältnis ist daher mit dem Parameter $\alpha$ festgelegt.

Die allgemeine Lösung wurde mit Hilfe einer IBM 7090 für verschiedene Parameterkombinationen $(\alpha, \beta)$ durchgeführt. Dabei war zu beachten, daß der Parameter $\beta$ wegen der Voraussetzung geradliniger Ionenbahnen auf den Bereich

$$
\beta \leqq\left(2 / x_{\mathrm{w}}\right)\left(l_{-} / R\right)\left(T_{0} / T_{-}\right) \sqrt{m_{+} / m_{-}}
$$

$\mathrm{zu}$ beschränken ist.

Die weiter erforderlichen Angaben sind von der Gasart abhängig und nur unzuverlässig bekannt.

4 G. Mierdel, Wiss. Veröff. Siemens 17, 277 [1938].
Für Helium finden wir in der Literatur ${ }^{4}$

$$
\begin{gathered}
\gamma=0,04, \quad A=2 \frac{p l_{-}}{\mathrm{cm} \text { Torr }}, \\
p l_{-}=0,0384(1+\Theta)^{3 / 2}[\mathrm{~cm} \text { Torr }] \\
\frac{E}{p}=\frac{198 \sqrt{\Theta}}{(1+\Theta)^{3 / 4}} \sqrt{\frac{1+2,6 \Theta}{e^{0,77 / \Theta}}}\left[\frac{V}{\mathrm{~cm} \text { Torr }}\right] .
\end{gathered}
$$

Die Resultate für die Ionen- und Elektronendichte und den Potentialverlauf sind in den Abb. 1 bis 3 und in Tab. 1 wiedergegeben.

Die $E / I$-Charakteristik ist in den Abb. 4 und 5 dargestellt. Abb. 6 zeigt den verwendeten Zusammenhang (26) zwischen Temperatur $T_{\text {- }}$ und Feldstärke $E^{4}$.

\section{Diskussion}

Da das Verhalten der stoßfreien Plasmasäule durch die Parameter $\alpha$ und $\beta$ eindeutig bestimmt ist, sind Entladungen mit gleichem $\alpha$ und $\beta$ ähnlich. Die numerische Auswertung läßt erkennen, daß die Rechnung LANGMUIRs im Grenzfall $(\alpha, \beta) \rightarrow 0$ ( ,normaler Bereich“ ohne Magnetfeld) korrekt ist. Mit wachsenden Parametern $\alpha$ und $\beta$ treten jedoch Abweichungen auf.

Mit zunehmendem $\alpha$, d. h. mit zunehmender Subnormalität bzw. abnehmendem Strom (s. Abb. 3), zeigt sich ein Absinken des Wandpotentials $\eta_{\mathrm{w}}$ und eine Verflachung der Potentialkurven. Gleichzeitig schnüren sich die Trägerdichteprofile ein. Der Erzeugungskoeffizient $1 / \tau$, der im wesentlichen wie $x_{\mathrm{w}}$ verläuft, steigt mit zunehmender Subnormalität an. Die $E / I$-Charakteristik ist daher fallend.

Mit zunehmendem $\beta$, d.h. mit abnehmender Elektronenbeweglichkeit oder zunehmendem Magnetfeld (s. Abb. 1 und 2), nimmt ebenfalls das Wandpotential $\eta_{\mathrm{w}}$ ab und verflachen sich die Potentialkurven. Die Trägerdichteprofile bauschen sich dagegen mit wachsendem Magnetfeld auf und der Erzeugungskoeffizient nimmt ab. Die $E / B$-Charakteristik ist daher ebenfalls fallend. Diese Effekte sind in der normalen Säule (Abb. 1) sehr schwach, steigen jedoch mit wachsender Subnormalität stark an (Abb. 2).

Die beschriebenen Erscheinungen sind physikalisch einleuchtend:

Das radiale elektrische Feld hat seine Ursache in der Forderung, daß der elektrische Strom zu der isolierten Wand verschwinden muß. Im „normalen Be-

\footnotetext{
1. Frauenklinik und Hebammenschule
} 
reich" ohne Magnetfeld sind etwa gleichviel Elektronen und Ionen in der Säule vorhanden, wobei die Elektronen sich jedoch ungleich schneller als die Ionen zur Wand bewegen. Damit dennoch beide Trägerarten in gleicher Zahl die Wand erreichen, ist ein starkes radiales elektrisches Feld erforderlich. Mit zunehmender Subnormalität sinkt die Elektronendichte gegenüber der Ionendichte, mit zunehmendem Magnetfeld sinkt die Elektronenbeweglichkeit. Beide Effekte müssen das radiale Feld schwächen.

Die Teilchenerzeugung $\int_{0}^{R}\left(n_{-} / \tau\right) 2 \pi r \mathrm{~d} r$ ist proportional zur Elektronenzahl $\int_{0}^{R} n_{-} 2 \pi r \mathrm{~d} r$ und zum Erzeugungskoeffizienten $1 / \tau$ und muß den Teilchenverlust zur Wand decken.

$$
\int_{0}^{R}\left(n_{c} n / \tau\right) 2 \pi r \mathrm{~d} r=n_{\mathrm{c}} n_{\mathrm{w}} \bar{v}_{-} / 4 .
$$

Die zentrale Dichte $n_{\mathrm{e}}$ beeinflußt die Beziehung (27) nicht und $\bar{v}_{-}$ist nur relativ schwach veränderlich. Der Erzeugungskoeffizient $1 / \tau$ muß also wachsen, wenn $n_{\mathrm{w}}$ (Wandverlust) wächst oder/und wenn das Elektronendichteprofil (Elektronenzahl) sich einschnürt. Da das axiale elektrische Feld $E$ mit dem Erzeugungskoeffizienten wächst, sind ebenfalls die Aussagen über die $E / I$ - bzw. die $E / B$-Charakteristik verständlich.

Der Vergleich unserer Resultate mit denen der stoßbestimmten Säule zeigt, daß im normalen Bereich eine formelmäßige Übereinstimmung erzielt werden kann, wenn man die Ionenbeweglichkeit $\mu_{+}=e /\left(v_{+} m_{+}\right)$des stoßbestimmten Falles durch die Größe $\mu_{+}=2 e \tau / m_{+}$ersetzt. Es gilt dann in beiden Fällen für die Elektronendichte

$$
n_{-}=n_{\mathrm{c}} \exp \left\{-\eta\left(1+\mu_{+} / \mu_{-}{ }^{*}\right)\right\}
$$

und für den Eigenwert

$$
\lambda=\sqrt{\frac{1+\mu_{+} / \mu_{-}^{*}}{\tau D_{\mathrm{a}}}} ; \quad D_{\mathrm{a}}=\left(k T_{-} / e\right) \mu_{+} .
$$

Da im stoßbestimmten Fall der Zahlwert von $\mu_{+}$im allgemeinen wesentlich größer ist als im stoßfreien Fall, wirkt sich ein bestimmtes Magnetfeld in der stoßbestimmten Säule stärker aus. Im subnormalen Bereich läßt sich ein Vergleich zwischen den Ergebnissen für die stoßfreie und stoßbestimmte Säule nicht durchführen, da für die stoßbestimmte Säule keine Resultate vorliegen.

\section{Anhang}

In Gl. (7) wurde für den Druck die skalare Approximation verwendet.

Der allgemeine Drucktensor ist gegeben durch

$$
p_{-}=n_{-} m_{-}\left(\begin{array}{lll}
\overline{W_{r}^{2}} & \overline{W_{r} W_{\varphi}} & \overline{W_{r} W_{z}} \\
\overline{W_{\varphi} W_{r}} & \overline{W_{\varphi}^{2}} & \overline{W_{\psi} W_{z}} \\
\overline{W_{z} W_{r}} & \overline{W_{z} W_{\varphi}} & \overline{W_{z}^{2}}
\end{array}\right),
$$

wo $W=v_{-}-\bar{v}_{-}$die auf die mittlere Geschwindigkeit bezogene Relativgeschwindigkeit angibt. In diesem Ausdruck kann man aus Symmetriegründen die Nichtdiagonal-Elemente vernachlässigen, solange die Driftgeschwindigkeiten klein gemessen an der mittleren thermischen Geschwindigkeit sind.

In unserer Anwendung interessieren wir uns ausschließlich für den Gradienten des Drucktensors und erhalten

$$
\nabla \cdot p_{-}=\nabla_{r} n_{-} m_{-} \overline{W_{r}^{2}} .
$$

Die beiden anderen Diagonalelemente entfallen wegen der Rotationssymmetrie und der axialen Homogenität.

Damit lautet die Impulsbilanz (8)

$$
\Gamma_{-r}=-n_{-} D_{-}\left[-\frac{e}{k T_{-}} \nabla_{r} U+\frac{\overline{m W_{r}^{2}}}{k T_{-}} \frac{\nabla_{r} n}{n_{-}}+\frac{\nabla_{r} m_{-} W_{r}^{2}}{k T_{-}}\right] .
$$

Die skalare Druckapproximation setzt in dieser Beziehung

$$
n_{-} m_{-} \overline{W_{r}^{2}}=n_{-} k T_{-} \text {. }
$$

Um die Konsequenzen dieser Vereinfachung (A 3) abzuschätzen, untersuchen wir das Moment $\bar{W}_{r}^{2}$, wie es sich mit Hilfe der „abgeschnittenen MAxweLL-Verteilung“

$$
F\left(W_{r}\right) \mathrm{d} W_{r}=\left\{\begin{array}{lll}
0 & ; & W_{r}<-\sqrt{\left(2 e / m_{-}\right)\left(U-U_{\mathrm{w}}\right)} \\
\sqrt{\frac{m_{-}}{2 k T_{-}} \exp \left\{-\frac{m W_{r}^{2}}{2 \pi k T_{-}}\right\} \mathrm{d} W_{r} ;} & W_{r}>-\sqrt{\left(2 e / m_{-}\right)\left(U-U_{\mathrm{w}}\right)}
\end{array}\right.
$$


berechnet. Diese „abgeschnittene Verteilung“ beschreibt die maximale Störung, die durch die Gegenwart der Wand verursacht werden kann. Man findet

$$
\frac{m_{-} \overline{W_{r}^{2}}}{k T_{-}}=\frac{1}{2}\left(1+\operatorname{erf} \sqrt{\frac{e\left(U-U_{\mathrm{w}}\right)}{k T_{-}}}\right)
$$

Damit folgt aus der Impulsbilanz (6) anstelle von (8) die Beziehung

$\Gamma_{-r}=-n_{-} D_{-}\left[a(\eta) \nabla_{r} \eta+b(\eta)\left(\nabla_{r} n_{-}\right) / n_{-}\right]$

mit

$a(\eta)=1-\sqrt{\left(\eta_{\mathrm{w}}-\eta\right) / \pi} e^{-\left(\eta_{\mathrm{w}}-\eta\right)}$

und

$$
b(\eta)=\frac{1}{2}\left(1+\operatorname{erf} \sqrt{\eta_{\mathrm{w}}-\eta}\right) .
$$

Verwendet man den Zusammenhang (A 7) anstelle von Gl. (8) in unserer Auswertung, so ergeben sich in den Endresultaten nur verschwindende Abweichungen.

Dieses Ergebnis ist physikalisch einleuchtend. In dem Bereich, wo die Korrekturen der MaxwelL-Verteilung wesentlich werden, ist die Elektronendichte gegenüber der Ionendichte bereits so stark abgesunken, daß sie die Raumladung nicht mehr beeinflußt.

Die numerische Integration wurde durchgeführt auf der IBM 7090 des Rheinisch-Westfälischen Insituts für Instrumentelle Mathematik. Wir danken Herrn SPAтsснек für seine Hilfe bei der Programmierung.

\title{
Über die Wechselwirkung eines Plasmastrahls mit einem achsensymmetrischen Magnetfeld
}

\author{
K. Ragaller \\ Elektrophysikalisches Institut der Technischen Hochschule München \\ (Z. Naturforschg. 21 a, 725-732 [1966] ; eingegangen am 3. März 1966)
}

\begin{abstract}
The interaction of a plasma jet with an axisymmetric magnetic field, is calculated in linear approximation for a periodic and for a convergent or divergent field configuration.

The solution depends on the $\mathrm{M}_{\mathrm{AcH}}$ number, the magnetic pressure number, and the magnetic Reynolds number.

In the convergent field, the plasma is decelerated. If the flow is supersonic, this is connected with a contraction of the jet leading beyond equilibrium and therefore being followed by an expansion. In the subsonic case, the stopping is accompanied by a broadening of the jet.

With increasing magnetic Reynouds number, "wakes" appear which extend upstream or downstream, depending on the values of the magnetic pressure number and the MacH number.
\end{abstract}

Das experimentelle Studium magnetogasdynamischer Effekte, die durch induzierte Ströme und Magnetfelder gekennzeichnet sind, steckt noch im Anfangsstadium, da die Leitfähigkeit, die Geschwindigkeit und die geometrische Ausdehnung von experimentell erzeugbaren Plasmen bisher noch nicht groß genug waren. In jüngerer Zeit entwickelte Plasmabrenner ${ }^{1-3}$ lassen solche Versuche jedoch aussichtsreich erscheinen.

Theoretische Untersuchungen, die sich mit der Plasmaströmung um Körper bei Anwesenheit eines Magnetfelds in linearer Näherung befassen ${ }^{4-6}$, zeig-

1 Th. Peters, Z. Naturforschg. 19 a, 1129 [1964].

2 K. Ragaller, Dissertation Techn. Hochschule München 1966.

3 A. C. Ducati, G. M. Giannini u. E. Muehlenberger, Am. Inst. Aeron. Astronaut. J. 2, 1452 [1964]. ten eine Fülle von neuen Lösungen auf. Diese Ergebnisse lassen sich aber vorerst nicht im Experiment nachprüfen, da mit unendlicher Ausdehnung und vielfach auch mit unendlicher Leitfähigkeit des Plasmas gerechnet wird.

Das Ziel der vorliegenden Arbeit ist es nun, eine experimentell relativ einfach realisierbare magnetogasdynamische Strömung theoretisch zu untersuchen. Als wohl einfachstes Problem dieser Art wird die Wechselwirkung eines Plasmastrahls mit einem achsensymmetrischen Magnetfeld in linearer Näherung behandelt.

4 W. R. Sears, Rev. Mod. Phys. 32, 701 [1960].

5 J. E. McCune u. E. L. Resler, J. Aerospace Sci. 27, 493 [1960].

${ }^{6}$ H. Grar, Rev. Mod. Phys. 32, 830 [1960]. 